\title{
Expanding use of osseointegrated implantation using 3-dimensional surgical planning: a paradigm shift in dental reconstruction
}

\author{
Peter J. Lancione ${ }^{1}$, Tony Satroplus ${ }^{2}$, Sasha Valentin ${ }^{3}$, Meade Van Putten ${ }^{3}$, Kyle K. VanKoevering ${ }^{1}$, Stephen \\ Y. Kang ${ }^{1}$, Matthew O. Old ${ }^{1}$, Nolan B. Seim ${ }^{1}$ \\ 'Department of Otolaryngology-Head and Neck Surgery, The Ohio State University Wexner Medical Center, Columbus, OH \\ 43210, USA \\ ${ }^{2}$ The Ohio State University College of Medicine, Columbus, OH 43210, USA. \\ ${ }^{3}$ Department of Dentistry-Maxillofacial Prosthodontics, The Ohio State University Wexner Medical Center, Columbus, OH \\ 43210, USA.
}

Correspondence to: Dr. Nolan B. Seim, Department of Otolaryngology-Head and Neck Surgery, James Cancer Hospital and Solove Research Institute, 460 West 10th Ave, Columbus, OH 43210, USA. E-mail: Nolan.Seim@osumc.edu

\begin{abstract}
How to cite this article: Lancione PJ, Satroplus T, Valentin S, Van Putten M, VanKoevering KK, Kang SY, Old MO, Seim NB. Expanding use of osseointegrated implantation using 3-dimensional surgical planning: a paradigm shift in dental reconstruction. Plast Aesthet Res 2021;8:13. http://dx.doi.org/10.20517/2347-9264.2021.02
\end{abstract}

Received: 07 Jan 2021 Accepted: 21 Jan 2021 Published: 9 Feb 2021

Academic Editor: Matthew Spector Copy Editor: Yue-Yue Zhang Production Editor: Xi-Jun Chen

\begin{abstract}
Medical modeling and 3-dimensional (3D) virtual surgical planning represent a rapidly expanding, technological advancement especially useful in complex mandibular or maxillary defects in head and neck reconstruction. With utilization of 3D surgical planning, the reconstructive surgeon can initiate dental rehabilitation during the primary surgery with osseointegrated implants $(\mathrm{Ol})$, streamlining a typically lengthy process to full oral rehabilitation. Careful patient selection is important to optimize outcomes with immediate OI, and factors to consider during the evaluation process include pathology, prognosis, anticipated defect, dental status, donor site availability, and patient motivation and resources. Synthesizing this information and developing a reconstructive plan with a multidisciplinary team approach is critical to expedite dental rehabilitation for select patients. A review of relevant literature and our surgical planning algorithm for selecting candidates for immediate $\mathrm{Ol}$ is provided, along with our experience using this decision algorithm in a uniquely complex clinical case.
\end{abstract}

Keywords: Dental reconstruction, 3-dimensional modeling, microvascular free tissue transfer, medical modeling, osseointegrated implant, virtual surgical planning 


\section{INTRODUCTION}

Mandibular and maxillary resections involve complex ablative procedures and are among the most challenging reconstructive cases in head and neck surgery. In these cases, the surgeon must contemplate both the oncologic and functional outcome along with quality of life and aesthetic considerations. In the past, the primary goals of free flap reconstruction were to ensure flap survival, replace tissue volume loss, create a safe wound, and provide structural support. With modern advances in microvascular free tissue transfer, flap survival now exceeds $95 \%{ }^{[1]}$. Increased success and efficiency of modern microvascular reconstructive surgery allows for the surgeon to think beyond filling a defect and expand the scope of the reconstruction with sophisticated approaches to maximize outcomes for both form and function; particularly incorporating expedited dental rehabilitation.

The use of a two-team free flap approach has become commonplace at many high-volume head and neck centers. This, along with the accessibility of a multidisciplinary team and 3-dimensional (3D) modeling, allows for a technologically advanced, collaborative approach for improved patient care. The incorporation of 3D modeling for head and neck surgery allows for improved accuracy for ablative approaches and, particularly, as a reconstruction template for the microvascular team. These $3 \mathrm{D}$ techniques are gaining momentum with success in both mandibular and maxillary reconstruction ${ }^{[2,3]}$. Along with these factors, the use of pre-surgical planning and modeling allows for the opportunity of immediate osseointegrated implants (OI) during the primary surgery. This can then streamline dental rehabilitation in select patient populations. First popularized by Levine et al. ${ }^{[4]}$, same day dental reconstruction is now attainable at select centers for a fraction of patients. The use of upfront OI offers several advantages, most notably a possibility for decrease time to complete dental reconstruction by at least 6 months. Alternatively, when delaying OI, the course to complete dental rehabilitation can be lengthy, taking on average 44 months ${ }^{[5]}$.

In this article, we focus on the details surrounding a decision for immediate OI as well as the clinical algorithm used for patient selection. Extensive multidisciplinary thought and planning on the front end of treatment must be used in assessing the expected defect and pathology, the patient's baseline dentition and goals, availability of donor sites, and expected surgical outcome along with potential need for adjuvant therapy. Synthesizing these factors and combining clinical and radiographic findings with medical modeling allows for a comprehensive surgical plan and can expand the use of $3 \mathrm{D}$ planning and immediate OI now and in the future.

\section{RECONSTRUCTIVE CONSIDERATIONS}

\section{Defect and pathology}

One of the main benefits of 3D surgical planning is knowing exactly where bony cuts will be made prior to making an incision, which improves surgical precision in mandible and maxillary ablative and reconstructive surgery ${ }^{[6-8]}$. For this reason, it is crucial to understand the lesion, pathology, and timing of surgery in order to anticipate the defect required for oncologic success. Benign lesions, due to slow growth and progression, are clearly preferred when attempting immediate OI. Moreover, in benign lesions, there is rarely a need for adjuvant therapy.

Postoperative radiation in the setting of malignancy brings additional risks and potential intermediate and long-term complications into consideration. One major risk is osteoradionecrosis. The need for adjuvant therapy, however, is not a strict contraindication. A recent review on this topic found the overall success rate of OI performed prior to initiation of radiation therapy was $67.4 \%$, compared to $93.1 \%$ in patients with OI performed greater that one year after completion of radiation ${ }^{[9]}$. These findings, however, may skew the success of delayed implantation, as patients who developed osteoradionecrosis or other complication would not likely be candidates for delayed OI. On the other hand, the success of primary implantation overall has 
been displayed by multiple comparative studies and cite success of up to $94 \%$ with immediate $\mathrm{OI}^{[10,11]}$. This highlights the need for a thorough preoperative evaluation algorithm for patient selection.

It is critical to note, the risk of immediate dental implant failure increases in a linear fashion with radiation dose. Unfortunately, in most circumstances, dose is based on final surgical pathology which is not available until after implantation has already been performed. Previously, Anderson et al. ${ }^{[12]}$ devised a guideline for dental implantation with radiation therapy, finding that OI placement either $>14$ days prior to or between 6 to 24 months after radiation is the ideal timeframe, and while doses at or above 50 Gy are preferred, doses greater than 70-75 Gy are inadvisable. One must of course weigh the increased time to dental rehabilitation with delayed implantation as well.

If immediate $\mathrm{OI}$ is desired in malignant cases, $3 \mathrm{D}$ modeling assist in other ways such as pre-planning surgical cuts. When doing so, it is important to plan for increases in tumor size during surgical scheduling. Plates are often created using 3D printing and can be extended well beyond the planned bony cut so that margins can be extended if dictated by the lesion and the planned reconstruction may still go forward with only minor alterations to the free flap. Another consideration for immediate OI is monitoring for recurrence and the possibility of needing any future resection. There is also the possibility of the unknown, such as a positive surgical margin, which increases the risk of recurrence and leads to higher doses of radiation. The risk of positive margin in bony tumors has historically been highest with sarcomas and high grade salivary gland carcinomas however can occur with any malignant lesion ${ }^{[13]}$. For this reason, aggressive local tumors are likely better suited for delayed OI after anticipated adjuvant therapy and an appropriate disease-free interval. Taking all this into account, however, in carefully selected patients with malignancy requiring adjuvant therapy, immediate OI can still be performed with good outcomes ${ }^{[14]}$.

On the other hand, benign lesions such as ameloblastomas, other dental lesions, and medically related osteonecrosis of the jaw may offer more predictable outcomes with OI. These lesions are typically slow growing giving a more definite defect size. Furthermore, the patients associated with these tumors tend to be younger, healthier, and with better dental health overall compared to malignant counterparts where chronic smoking remains the highest risk factor for disease and likely impacts the overall success of reconstruction. Typically, dental reconstruction is a significant goal for patients in this setting as well.

\section{Donor site availability}

An assessment of available donor sites is a vital step in planning mandibular and maxillary reconstruction. Many factors impact the ability to utilize different donor sites for reconstruction. This includes nature of anticipated defect, availability of donor vessels and associated need for pedicle length, history of prior surgeries, lower extremity trauma, peripheral vascular disease, and patient body habitus. At our institution, nearly all patients requiring significant osseous reconstruction undergo CT angiogram of the lower extremities to assess the vascular anatomy and donor site viability of the fibula as it remains the work-horse option due to superior bone stock compared to other options. The CT also allows the team to assess the quality of the fibula bone in the virtual environment and develop patient specific plans. MR angiograms do not allow for this planning capability.

When considering immediate OI, the fibula typically serves as a reliable bony construct to hold an implant. Fundamentally, the fibula offers a 360-degree cortical bone stock, providing increased strength and integrity at any orientation, while all other bony donor sites are partial bone grafts that are deficient of cortical bone on at least one surface. Still, despite its advantages, many patients are unable to tolerate this flap due to vascular anatomy, body habitus, or other co-morbidity. For this reason, the scapular tip, lateral scapula and osteocutaneous radial forearm flaps have become more frequently used in recent years for bony reconstruction; however, in the case of immediate OI, these flaps are far less ideal. 
Previous reports confirm, the fibular osteocutaneous free flap is typically necessary for immediate insertion of $\mathrm{OI}^{[11,15]}$. With 12-15 $\mathrm{mm}$ of bone height and bicortical bone, the fibula is most receptive to endosteal implantation ${ }^{[16]}$. The bone height, length, low donor site morbidity in appropriate patients, and physical distance from the primary surgical site allowing for convenient harvest ${ }^{[17]}$. For these anatomic reasons, the fibula flap is clearly the first choice for immediate OI and even preferred for delayed OI as well. That being said, other osteocutaneous flaps can be used for delayed OI but the ability to use the fibula donor site remains a key main decision point in choosing immediate versus delayed OI.

In cases where lower extremity vascular anatomy or other issue precludes the use of the fibula free flap, the scapula has become a popular option for bony reconstruction of oral-maxillofacial defects. The scapula bone provides $10-14 \mathrm{~cm}$ of bone with adequate depth to support OI at multiple segments in males. However, typically the female scapula has only enough bone to support implantation at the proximal portion of the scapula ${ }^{[18]}$. The scapula tip free flap is another option for mandibular and midface reconstruction and has been found to support dental implantation in up to $95 \%$ of cases based on imaging review by independent oral surgeons ${ }^{[19]}$. In these cases, a CT chest for $3 \mathrm{D}$ surgical planning is needed if OI is desired immediately or in a delayed fashion in order to plan the most appropriate bone for reconstructive purposes.

Execution of immediate OI with a scapula reconstruction is technically much more difficult than a fibula, however. The extensive muscle surrounding the bone, the position of the vascular pedicle, and the presence of the cartilaginous tip make use of cutting guides a challenge. Additionally, the extensive muscle around the bone makes accurate implant placement difficult and fraught with a higher margin for error. Despite this, $3 \mathrm{D}$ modeling is beneficial in planning scapula and scapular tip reconstructions. In these cases, the reconstructive surgeon typically may have to "free-hand" the bony osteotomies to a custom plate and plan for OI after bony union has occurred ${ }^{[20]}$. Similarly, in situations when an osteocutaneous radial forearm flap is used, delayed OI is needed as the radius bone needs to be double-barreled or the bone stock may need to be re-enforced at a later date to hold an implant ${ }^{[2]]}$.

\section{Evaluation of patient dentition}

The patient's existing dentition and functional goals can greatly influence reconstructive planning. Many patients in the head and neck cancer population present with compromised dentition and risk factors of long-term tobacco and alcohol use, all of which may complicate dental rehabilitation. Poor dental health remains a relative contraindication to OI. Due to the need for ongoing dental care following implantation, these patients are likely to be better served with denture appliances. In the case of atrophic mandible in elderly and totally edentulous patients, this can severely limit meaningful dental rehabilitation as a primary goal as well ${ }^{[5]}$. The elderly or edentulous patient with atrophic mandible can lack the bone volume necessary for $\mathrm{OI}$ in the native mandible and may not have sufficient alveolar ridge height to support traditional removable dentures complicating dental rehabilitation options. In these patients, teams must remain openminded and discuss other potential rehabilitation options.

Prior to primary surgery, the initial maxillofacial prosthodontic evaluation of the patient's existing dentition is of upmost importance. With the proper preoperative evaluation, selecting patients with good baseline dentition, lack of high-risk lifestyle habits, and suitable oral hygiene will increase the chance of success with immediate OI. The patient's motivation for achieving full oral rehabilitation is an important factor to assess as well. Then, once appropriately selected, assessment of occlusion, skeletal relationships, and dental position determines the vertical and horizontal position of the bony flap and determines the position for planned implant placement. 


\begin{tabular}{lccc}
\hline & Insurance coverage & Patient cost & Medical modeling \\
\hline Removable dental prosthesis & Yes & $\$$ & No \\
Removable prosthesis affixed by OI & Possible & $\$ \$$ & For Immediate OI \\
Fixed prosthesis with complete OI & Not typical & $\$ \$ \$$ & Typically required \\
\hline
\end{tabular}

Individual patient cost is determined by factors including insurance coverage for the procedure, the initial cost of medical modeling $(\mathrm{MM})$, and the long-term savings with immediate implantation reducing the number of surgeries for the patient. MM typically adds $\$ 7,000-\$ 10,000$ to the upfront cost of the surgery; however. this is not necessarily a cost to the patient[21]. \$ - removable dental prosthesis typically covered by insurance and no need for $\mathrm{MM}$ with minimal to no patient costs; $\$$ - potential for insurance coverage for removable prosthesis; cost of $\mathrm{MM}$ and OI can be bundled into primary surgery if performed upfront; $\$ \$ \$$ - immediate Ol can be bundled into primary surgery however fixed prosthesis are not covered by insurance and incurs a significant out-of-pocket cost. \$: Removable dental prosthesis typically covered by insurance and no need for MM with minimal to no patient costs; $\$$ : potential for insurance coverage for removable prosthesis; cost of $\mathrm{MM}$ and $\mathrm{Ol}$ can be bundled into primary surgery if performed upfront; $\$ \$$ : immediate Ol can be bundled into primary surgery however fixed prosthesis are not covered by insurance and incurs a significant out-of-pocket cost; Ol: osseointegrated implants.

\section{Dental rehabilitation options and cost implications}

Overall medical expenses and patients' out-of-pocket cost related to medical modeling and 3D planning can be significant and are frequently discussed around this topic. First to consider, there are three main options for oral rehabilitation [Table 1]: a conventional removable resection prosthesis, a removable mandibular resection prosthesis assisted by $\mathrm{OI}(\mathrm{s})$, and a fixed resection prosthesis completely supported by OIs. As the number of implants increases the cost, the complexity of the prosthetic rehabilitation also increases. This includes an increase in the time and complexity of the pre-planning sessions. A conventional removable resection prosthesis is considered a medical device and is usually covered by medical insurance. A removable resection prosthesis assisted by implants would add additional cost to the rehabilitation and the implants may or may not be covered by their insurance. The fixed resection prosthesis completely supported by implants is the most-costly option since the final, fixed prosthesis is not covered by the patient's medical insurance. Regardless of option, it is important to maintain open lines of communication between all of the providers involved in order to optimize the reconstruction for the particular rehabilitation choice.

Regarding direct patient costs, this is a difficult topic to discuss in general terms as each patient's situation, hospital environment, and practice patterns are unique and coverage is highly variable among insurers. However, immediate OI, at the time of primary surgery, allows for the OIs to be bundled into the surgery, same as the reconstruction plate and screws, and thus typically does not add a cost to the individual patient. The outpatient costs for definitive dental reconstruction are variable. In the case of removable dental prostheses, these are often covered by insurance. More traditional dental implants are most often an out-of-pocket cost. These issues and potential obstacles must be taken in consideration and discussed with each patient and this will help guide the final rehabilitation choice.

In regard to overall expense, one must always consider the need for the advantages afforded by 3D surgical planning and balance this with the known increase in operative planning and cost for each case. In the current setting of rising healthcare expenditures and cost-conscious medical care in the United States, the expanded use of medical modeling technologies can present an impasse for the reconstructive surgeon. A cost-effectiveness analysis by Fatima et al. ${ }^{[22]}$ found that although virtual surgical planning can reduce total operative time and ischemia time, this does not lead to reduction of downstream complications or improve outcomes. On the other hand, more recent studies have supported the time savings, improved accuracy, and equal cost of $3 \mathrm{D}$ surgical planning in head and neck reconstruction ${ }^{[2,24]}$. Though the use of 3D modeling can add $\$ 7,000-\$ 10,000$ to upfront surgical costs ${ }^{[22]}$, in the end these expenses may be offset by decreased operative time and length of stay ${ }^{[8,25]}$. Additionally, the added cost of medical modeling is only a fraction of the overall cost of surgery and is typically bundled into the price of the procedure and postoperative 
hospitalization.

To address cost concern and improve patient outcomes, many medical centers have developed their own 3D printing capabilities. Large academic centers or health systems with affiliated engineering schools may have the resources, personnel, and experience to execute many of the modeling and planning protocols internally. A coordinated effort with commercial plating companies and engineers may allow for a more cost-efficient model. As with all technological advances, the costs are typically higher at the beginning and decrease with increased utilization and improved, more efficient care models. Certainly, this may be the case regarding future use of this technology as well.

\section{Obstacles in dental rehabilitation}

As discussed previously, many factors may preclude the use of 3D surgical planning and immediate OI for dental rehabilitation. Discussions leading up to surgery not only include collaboration across specialties, but also involve the patient in their care. The decision to undergo reconstruction with immediate OI is often based on the goal of an expedited dental rehabilitation and return to normal function. As such, it is important to manage patient expectations and make clear the risks and potential complications in addition to postoperative care required. With immediate OI, strict adherence to an oral hygiene regimen is required in the early postoperative period to minimize the risk of complications and improve long-term success. It is, therefore, of the utmost importance to properly screen patients and assess their ability to follow through with the outlined oral care plan.

The patient must be highly motivated for the best chance of successful dental rehabilitation. The head and neck cancer population, having higher rates of potentially complicating factors including smoking, malnutrition, and xerostomia, must all be taken into account. Whether a limiting factor exists or not, there are many traditional options to restore patient dentition. In the correct setting, this approach can provide an optimal functional result without the use of OI.

This approach must be weighed, of course, with the fact that traditional dental reconstructive options can pose difficulties in their own right postoperatively ${ }^{[5,26]}$. Removable dentures rely on the remaining alveolar ridge to remain fixed in place, and these structures are invariably disrupted and can lead to instability of the appliance. The alteration of the gingivolabial sulcus, as well, can pose difficulties fitting and creating stability for a prosthesis. Therefore, at times, a well-selected patient may benefit from immediate OI in order to stabilize a planned, removable prosthesis as well.

\section{CLINCAL CASE}

There are many instances when 3D planning optimizes the outcomes in complex head and neck surgery. However, its use for a packaged resection and reconstructive approach for even the most challenging of cases may improve the oncologic, functional, and economic outcomes for well selected patients. For this reason, even in challenging reconstructive cases, patient that fit one's multi-disciplinary algorithm for immediate OI should be considered [Figure 1]. We present a unique and representative case in this regard.

\section{Atypical case for immediate osseointegrated implantation}

A 69-year-old female, with a past medical history significant for chronic kidney disease, hypertension, osteoporosis, well controlled type-2 diabetes, and hepatitis C, presented with stage III bisphosphonaterelated osteonecrosis of the jaw (BRONJ). Of note, she had a 5-year history of alendronic acid use to treat her osteoporosis as well as a distant history of mandibular dental post implantation to secure her lower denture. Prior to presentation to otolaryngology, she experienced local infection with abscess formation requiring combined intraoral and extraoral incision and drainage. At that time, CT scan revealed necrosis of the mandible consistent with BRONJ based on her medical history. She then underwent anterior 


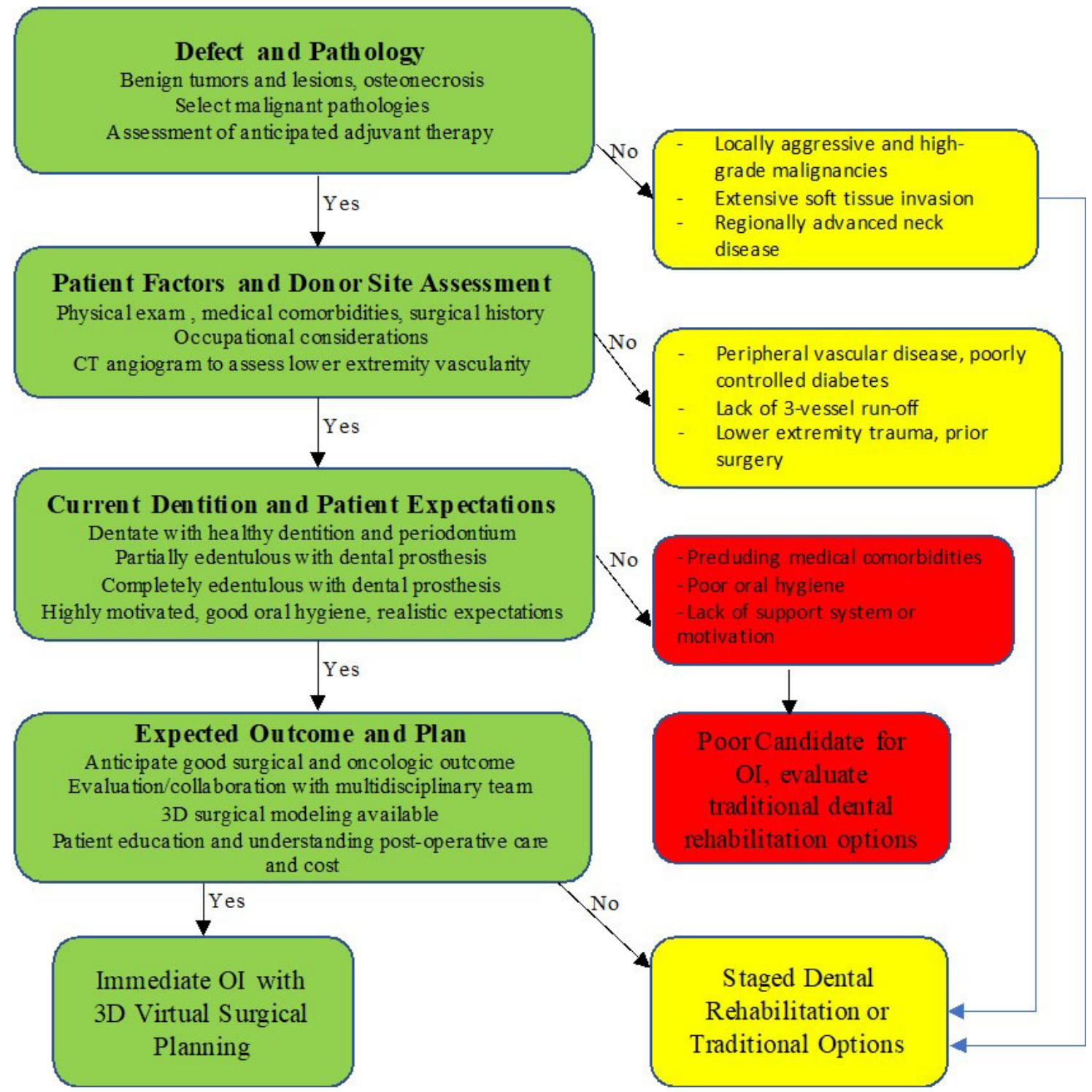

Figure 1. Reconstructive algorithm. Reconstructive algorithm used in evaluating feasibility of immediate osseointegrated implants for dental rehabilitation in planned maxillectomy and mandibulectomy.

mandibulectomy and plate-only reconstruction with oral and maxillofacial surgery and was referred to Otolaryngology-Head and Neck Surgery clinic for evaluation six months after this initial surgical resection. Clinically, the patient examination revealed completely edentulous status wearing a complete maxillary denture fixed with denture adhesive. The patient's prior segmental mandible resection created a defect from the right mandibular body to the left mandibular body with now scarred and retracted oral vestibular mucosa healed intact and without fistula.

The options for dental rehabilitation were reviewed with our maxillofacial prosthodontic team, and due to the nature of her defect and goals of reconstruction, OI was recommended. We discussed delayed versus immediate OI with the patient who opted for $3 \mathrm{D}$ medical modeling and immediate implantation. A CT angiogram of the lower extremities was performed which revealed three vessel runoff bilaterally. CT neck with contrast was performed to assess for free flap donor vessels. A fine cut CT facial was obtained [Figure 2] and patient-specific MM (KLS Martin Group, Jacksonville, FL) was utilized by the head and 

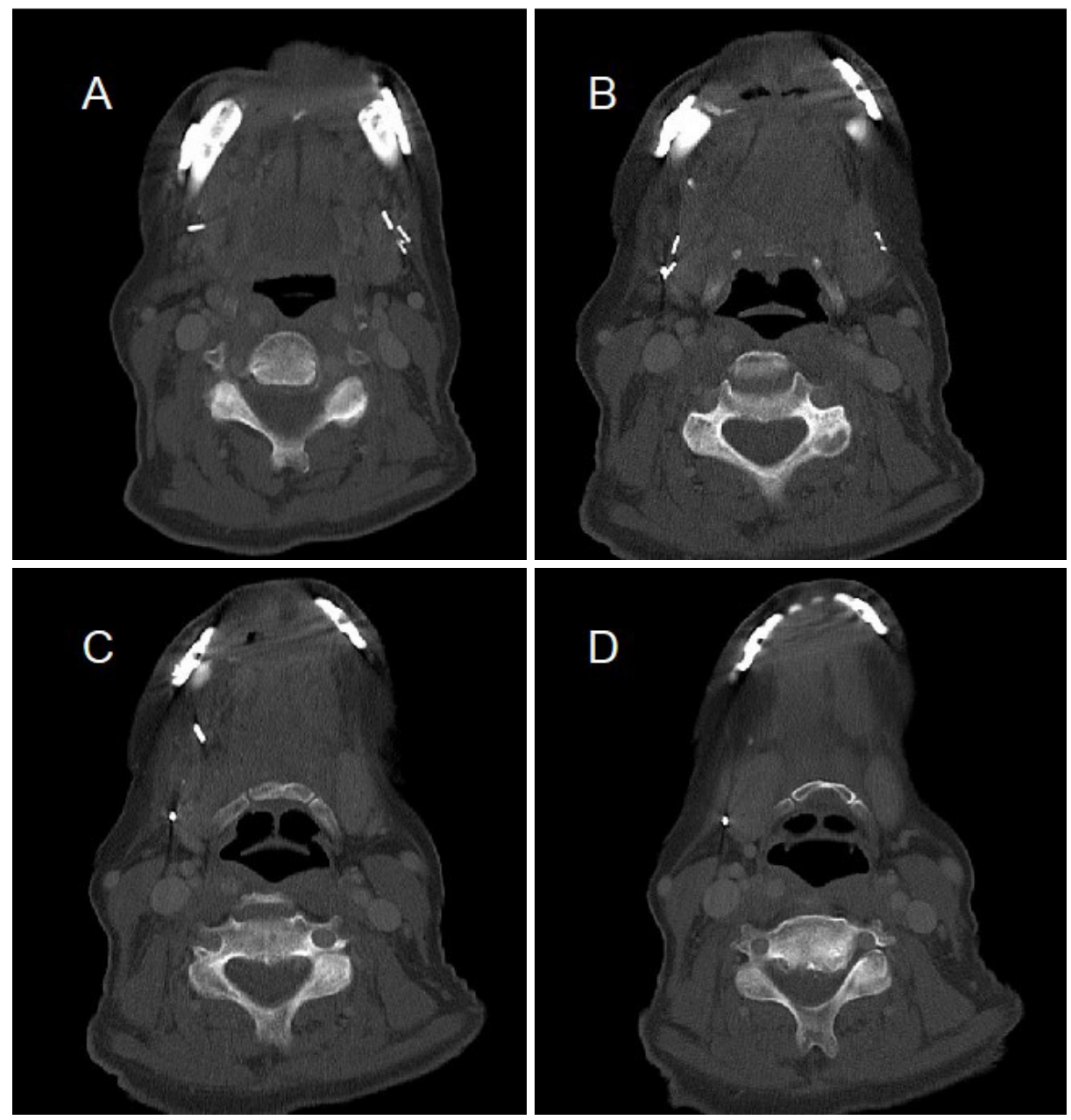

Figure 2. Preoperative imaging. CT facial, axial images display extent of prior anterior mandibulectomy $(A, B)$ and protrusion of plateonly reconstruction (C, D).

neck reconstructive surgeon and maxillofacial prosthodontist accounting for patient-specific anatomy and contour of the fibula. MM was then used to virtually plan the surgical resection and reconstruction, in which cutting guides for the mandible and fibula were generated [Figure 3].

The patient underwent surgery with planned left fibula free flap. KLS 3D printed cutting guides were placed on the fibula and OI holes were drilled while the fibula remained perfused in the leg. Pre-planned osteotomies were then made, and the 3D custom pre-bent $2.5 \mathrm{~mm}$ mandibular reconstruction plate was then secured to the fibula. Implants and healing abutments were then placed into the drill holes [Figure 4]. The free flap was then harvested and attached to the mandibular defect and flap vessels anastomosed to the right facial artery and right external jugular vein. Postoperatively the patient did well and was discharged from the hospital on postoperative day five with a full oral diet. No tracheostomy was indicated as there was no intra-oral mucosal defect.

\section{Discussion}

Due to the difficult situation in which this patient presented, missing the anterior segment of the mandible, non-anatomic plate in place, completely edentulous status, and desire to return to full oral diet, all reconstructive options were considered with particular attention placed on long-term dental rehabilitation. The use of $3 \mathrm{D}$ modeling and pre-surgical planning in a multi-disciplinary format allowed our team to 


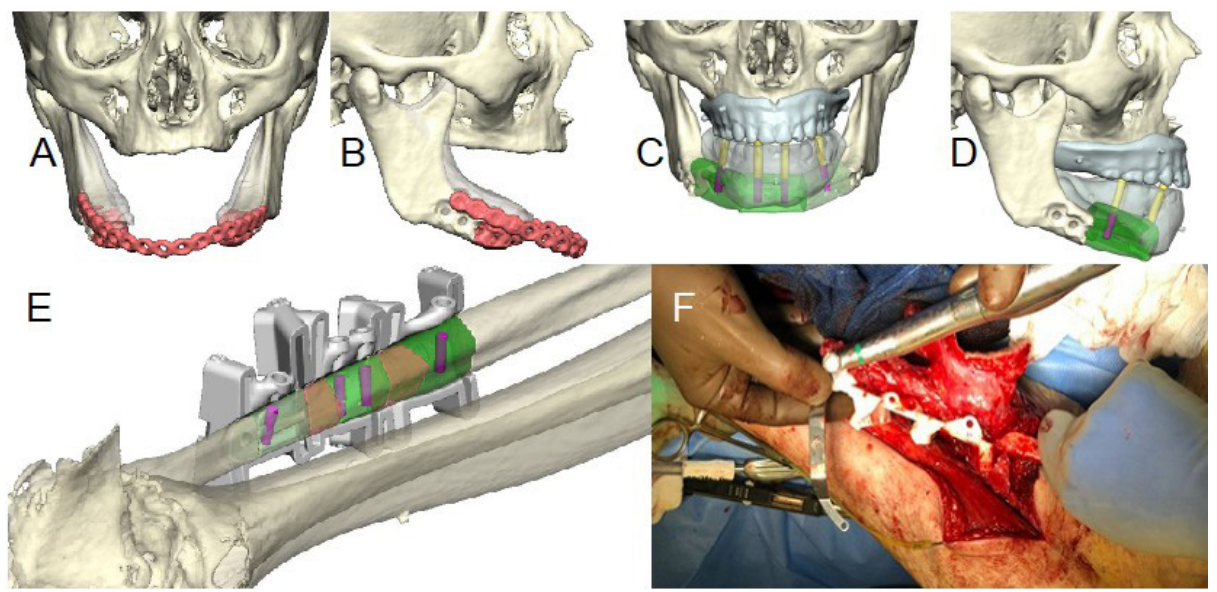

Figure 3. 3D medical modeling. Patient specific medical modeling for planned mandibular reconstruction. Coronal (A) and right lateral (B) view of previous segmental mandibulectomy with plate-only reconstruction. 3D modeling of planned left fibula free flap reconstruction, including implants and healing caps in coronal (C) and right lateral (D) views. KLS 3D printed cutting guides modeled to patient's left fibula (E) and placed on fibula for OI hole drilling (F).
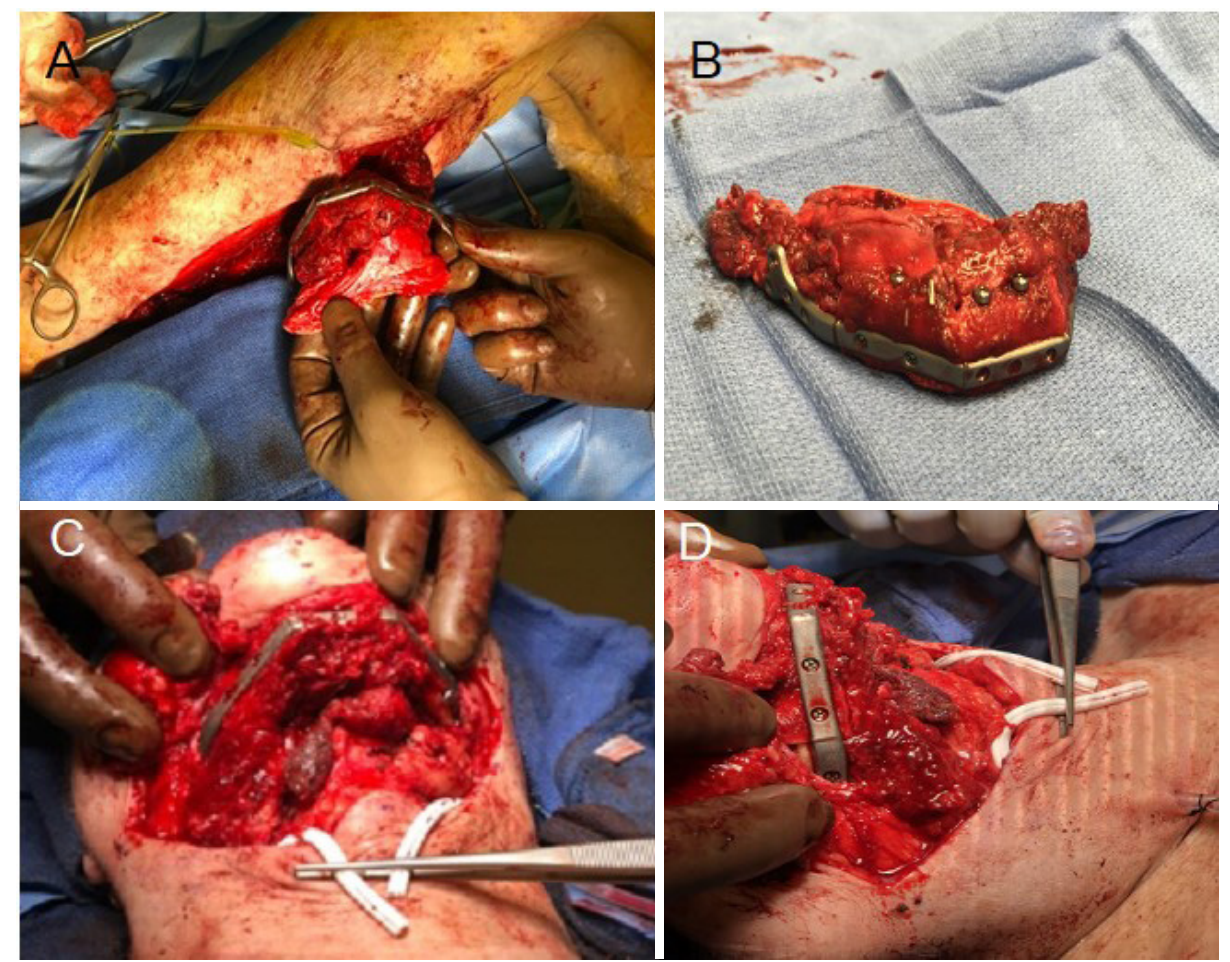

Figure 4. Free flap customization. Inset of fibula free flap after pre-planned osteotomies, osseointegrated implants inset, and plate attachment. (A) Fibula fastened to pre-bent $2.5 \mathrm{~mm}$ mandibular reconstruction plate. (B) Implants and healing caps placed into drill holes of free flap. Mandibular reconstruction plate, fibula, and implants placed to repair defect from inferior (C) and right lateral views (D).

re-design the mandibular shape and size and plan the position of OIs. The favorable factors in her case include a relatively small defect of known size and lack of malignant etiology. With a benign pathology, the chance of needing to extend bony cuts is removed and the risks associated with postoperative radiation are eliminated. The patient's CT angiography and lower leg anatomy were favorable for a fibula free flap reconstruction, thus allowing the possibility for immediate OI. After the dental reconstruction plan was 
considered, and the patient was interested in the upfront approach, bundling the OI into the primary surgery offered the opportunity to decrease the overall time to full oral rehabilitation and bundle the implant cost into the primary surgery. As such, using an algorithm [Figure 1] and synthesizing patientspecific information in a multidisciplinary approach can lead to a well-defined surgical plan and successful outcomes with immediate OI.

\section{CONCLUSION}

Osseus free flap reconstruction of the mandible and maxilla requires proper planning, patient preparation, and collaboration to achieve desirable functional and aesthetic outcomes. The emergence of MM and 3D surgical planning makes immediate OI for expeditated full dental rehabilitation not only possible, but an ideal option for the appropriately selected patient. When considering immediate implantation, we recommend utilization of a thorough, personalized, pre-surgical plan in a multidisciplinary approach with the goals of reconstruction well aligned with the patient's goals and expectations.

\section{DECLARATIONS}

\section{Authors' contributions}

Made substantial contributions to conception and design of the study, performed data analysis and interpretation, drafting the work or revising it critically for important intellectual content, final approval of the version to be published, and agreement to be accountable for all aspects of the work in ensuring that questions related to the accuracy or integrity of any part of the work are appropriately investigated and resolved: Lancione PJ, Satropolus T, Seim NB

Made substantial contributions to conception and design of the study, drafting the work or revising it critically for important intellectual content, final approval of the version to be published, and agreement to be accountable for all aspects of the work in ensuring that questions related to the accuracy or integrity of any part of the work are appropriately investigated and resolved: Valentin S, Van Putten M, VanKoevering KK, Kang SY, Old MO

\section{Availability of data and materials}

Not applicable.

\section{Financial support and sponsorship}

None.

\section{Conflicts of interest}

All authors declared that there are no conflicts of interest.

\section{Ethical approval and consent to participate}

Not applicable.

\section{Consent for publication}

Not applicable.

\section{Copyright}

(c) The Author(s) 2021.

\section{REFERENCES}

1. Gusenoff JA, Vega SJ, Jiang S, et al. Free tissue transfer: comparison of outcomes between university hospitals and community hospitals. Plast Reconstr Surg 2006;118:671-5.

2. Metzler P, Geiger EJ, Alcon A, Ma X, Steinbacher DM. Three-dimensional virtual surgery accuracy for free fibula mandibular 
reconstruction: planned versus actual results. J Oral Maxillofac Surg 2014;72:2601-12.

3. Kääriäinen M, Kuuskeri M, Gremoutis G, Kuokkanen H, Miettinen A, Laranne J. Utilization of three-dimensional computer-aided preoperative virtual planning and manufacturing in maxillary and mandibular reconstruction with a microvascular fibula flap. $J$ Reconstr Microsurg 2016;32:137-41.

4. Levine JP, Bae JS, Soares M, et al. Jaw in a day: total maxillofacial reconstruction using digital technology. Plast Reconstr Surg 2013;131:1386-91.

5. Petrovic I, Rosen EB, Matros E, Huryn JM, Shah JP. Oral rehabilitation of the cancer patient: a formidable challenge. J Surg Oncol 2018;117:1729-35.

6. Roser SM, Ramachandra S, Blair H, et al. The accuracy of virtual surgical planning in free fibula mandibular reconstruction: comparison of planned and final results. J Oral Maxillofac Surg 2010;68:2824-32.

7. Hirsch DL, Garfein ES, Christensen AM, Weimer KA, Saddeh PB, Levine JP. Use of computer-aided design and computer-aided manufacturing to produce orthognathically ideal surgical outcomes: a paradigm shift in head and neck reconstruction. J Oral Maxillofac Surg 2009;67:2115-22.

8. Chang EI. Long-term operative outcomes of preoperative computed tomography-guided virtual surgical planning for osteocutaneous free flap mandible reconstruction. Plast Reconstr Surg 2016;138:559e-60e.

9. Koudougou C, Bertin H, Lecaplain B, et al. Postimplantation radiation therapy in head and neck cancer patients: Literature review. Head Neck 2020;42:794-802.

10. Menapace DC, Van Abel KM, Jackson RS, Moore EJ. Primary vs secondary endosseous implantation after fibular free tissue reconstruction of the mandible for osteoradionecrosis. JAMA Facial Plast Surg 2018;20:401-8.

11. Jackson RS, Price DL, Arce K, Moore EJ. Evaluation of clinical outcomes of osseointegrated dental implantation of fibula free flaps for mandibular reconstruction. JAMA Facial Plast Surg 2016;18:201-6.

12. Anderson L, Meraw S, Al-Hezaimi K, Wang HL. The influence of radiation therapy on dental implantology. Implant Dent 2013;22:31-8.

13. Schusterman MA, Harris SW, Raymond AK, Goepfert H. Immediate free flap mandibular reconstruction: significance of adequate surgical margins. Head Neck 1993;15:204-7.

14. Tamaki A, Seim NB, Valentin S, et al. The use of medical modeling in microvascular free tissue transfer reconstruction with osseointegrated implantation in complex midface defects. Oral Oncol 2020;110:104982.

15. Hidalgo DA. Fibula free flap: a new method of mandible reconstruction. Plast Reconstr Surg 1989;84:71-9.

16. Moscoso JF, Keller J, Genden E, et al. Vascularized bone flaps in oromandibular reconstruction. A comparative anatomic study of bone stock from various donor sites to assess suitability for enosseous dental implants. Arch Otolaryngol Head Neck Surg 1994;120:36-43.

17. Okay DJ, Buchbinder D, Urken M, Jacobson A, Lazarus C, Persky M. Computer-assisted implant rehabilitation of maxillomandibular defects reconstructed with vascularized bone free flaps. JAMA Otolaryngol Head Neck Surg 2013;139:371-81.

18. Solis RN, Mahaney J, Mohhebali R, et al. Digital imaging evaluation of the scapula for prediction of endosteal implant placement in reconstruction of oromandibular defects with scapular free flaps. Microsurgery 2019;39:730-6.

19. Blumberg JM, Walker P, Johnson S, et al. Mandibular reconstruction with the scapula tip free flap. Head Neck 2019;41:2353-8.

20. Marchiano E, Stevens JR, Liao E, et al. Three-dimensional modeling of the scapular tip for anterolateral and lateral mandibular defects. Oral Oncol 2020;107:104718.

21. Gonzalez-Castro J, Petrisor D, Ballard D, Wax MK. The double-barreled radial forearm osteocutaneous free flap. Laryngoscope 2016;126:340-4.

22. Fatima A, Hackman TG, Wood JS. Cost-effectiveness analysis of virtual surgical planning in mandibular reconstruction. Plast Reconstr Surg 2019;143:1185-94.

23. Chen Z, Mo S, Fan X, You Y, Ye G, Zhou N. A Meta-analysis and systematic review comparing the effectiveness of traditional and virtual surgical planning for orthognathic surgery: based on randomized clinical trials. J Oral Maxillofac Surg 2020:S0278-2391(20)31111-3.

24. Mazzola F, Smithers F, Cheng K, et al. Time and cost-analysis of virtual surgical planning for head and neck reconstruction: A matched pair analysis. Oral Oncol 2020;100:104491.

25. Avraham T, Franco P, Brecht LE, et al. Functional outcomes of virtually planned free fibula flap reconstruction of the mandible. Plast Reconstr Surg 2014;134:628e-34e.

26. Curtis TA, Cantor R. The forgotten patient in maxillofacial prosthetics. J Prosthet Dent 1974;31:662-80. 